\title{
COMPARATIVE STUDY OF TERBINAFINE HYDROCHLORIDE TRANSFERSOME, MENTHOSOME AND ETHOSOME NANOVESICLE FORMULATIONS VIA SKIN PERMEATION AND ANTIFUNGAL EFFICACY \\ BY
}

\author{
Alaa A. Zaky ${ }^{* 1}$ and Mahmoud M. Tawfick ${ }^{2}$ \\ FROM \\ ${ }^{1}$ Department of Pharmaceutics and Industrial Pharmacy, Faculty of Pharmacy, Al-Azhar \\ University, Nasr City, Cairo, Egypt. \\ ${ }^{2}$ Microbiology and Immunology Department, Faculty of Pharmacy, Al-Azhar \\ University, Nasr City, Cairo, Egypt
}

\begin{abstract}
The purpose of the present research was to compare the skin permeation and study the antifungal efficacy of Terbinafine hydrochloride (TH) transfersome, menthosome and ethosome formulations under non-occlusive conditions. Terbinafine hydrochloride is an antifungal drug for onychomycosis. Poor permeability of its external preparation leads to poor curative effect. Preparation of $\mathrm{TH}$ transfersome utilized the mixture component model to determine the optimized desirable formula using different concentrations of nonionic surfactant, Span and Tween $\left(X_{1} \& X_{2}\right.$ respectively) were investigated. The results revealed that both $X_{1}$ and $X_{2}$ had a pronounced effect on vesicle size $\left(\mathrm{Y}_{1}\right)$ and entrapment efficiency $(\mathrm{EE})$ of the drug $\left(\mathrm{Y}_{2}\right)$. The vesicles were prepared and characterized for shape, size, zeta potential and entrapment efficiency. Ex vivo study via Franz diffusion cells was used for the percutaneous absorption studies. The optimum desirable formula of transfersome, menthosome and ethosome formulations were showed vesicle size (78.7, 122.8 and 67.8 $\mathrm{nm})$, zeta potential $(-8.28,8.77$ and $11.30 \mathrm{mV}$ ) and encapsulation efficiency (92.67, 93.86 and $95.75 \%$ ), respectively. Ex vivo permeation of the drug-loaded nanovesicle showed more than two to three folds higher permeation rate compared with drug suspension. Deformability verified elasticity of the preparation. Finally, TH nanovesicle formulations improved drug delivery with greater improvement in skin permeation and antifungal activity. Our selected formulae showed potent antifungal effect against Aspergillus niger for the treatment of fingernails or toenails.
\end{abstract}

Keywords; Terbinafine Hydrochloride, Transfersome, Menthosome, Ethosome, Nanovesicles

\section{Introduction}

Transdermal drug delivery offers many advantages over other routes of administration, including avoidance of first-pass metabolism and targeting of the active ingredient for a local effect (El Maghraby et al., 2008). Unfortunately, the formidable barrier characteristics of stratum corneum present a significant obstacle for most drugs to be delivered into and through it. To overcome the barrier, lipid vesicles have been proposed. Liposomes were first reported as carriers for topical use with lack of permeability of drugs to deeper skin layers (Mezei and Gulasekharam, 1980). Recently, vesicle drug delivery forms like niosomes, transfersomes, menthosome and ethosome 
provided several advantages. They are able to enhance drug bioavailability via enhancement of drug permeation and interactions with human skin, as well as prolongation of drug action and reduced administered drug toxicity (Dragicevic-Curic et al., 2010; Kumar et al., 2012; Mahale et al., 2012; van den Bergh et al., 2001)

Transferosomes are the first generation of elastic vesicles, which were first introduced in the early 1990s (Cevc and Blume, 1992). Its main compositions are phospholipids (membrane) and surfactant (membrane softening agent). Transfersomes the most important feature to prepared elastic vesicle caused by the presence of the edge activator. These elastic vesicles are able to squeeze through intercellular regions of the stratum corneum under the influence of the transepidermal water-activity gradient. Phospholipid (PL) hydrophilicity leads to xerophobia (tendency to avoid dry surroundings) thus, for the vesicles to remain maximally swollen on skin surface. The elasticity and flexibility of this vesicle minimizes the possibility of its rupture especially when applied onto the skin (Malakar et al., 2012).

The characters of nano-vesicle affected by edge activator types such as nonionic surfactant (Tween 80 and Span 80) having different HLB values (15 and 4.5 respectively). The vesicle prepared using Tween showed more deformability with less entrapment efficiency, this due to highly flexible and nonbulky hydrocarbon chains. In contrast, the vesicle containing span showed least deformability and high entrapment efficiency. These could be attributed to the highly hydrophobicity that reduced the formation of transient hydrophilic holes, hence, minimizing the amphiphilic property of the bilayers responsible for membrane fluidity. Transfersomes used as a carriers for various drugs such as analgesics, anesthetics, corticosteroids (Cevc et al., 1997; Essa et al., 2004), proteins such as insulin, albumin and sex hormones (Cevc, 2003; Cevc et al., 1995; Planas et al., 1992) and anticancer drugs (Hiruta et al., 2006; Trotta et al., 2004).

While, menthosome it is contain edge activator almost cationic surfactant (cetrimide). An edge activator is often a single-chain surfactant, having a high radius of curvature that destabilizes lipid bilayers of the vesicles and increases deformability of the bilayers (Duangjit et al., 2012). In addition, ethosome is phospholipid-based elastic nanovesicles containing a high content of ethanol (20-45\%). Ethosomal systems are much more efficient in delivering substances to the skin in the terms of quantity and depth, than either conventional liposomes or hydroalcoholic solutions.

Terbinafine (TH) is one of the most widely used antifungal drugs clinically (Lee et al., 2008). Onychomycosis is a fungal infection of the fingernails or toenails that causes discoloration, thickening and separation from the nail bed. This disease occurs in $10 \%$ of the general population but is more common in older adults. This higher prevalence in older adults is related to peripheral vascular disease, immunologic disorders or diabetes mellitus (Westerberg and Voyack, 2013). Onychomycosis is mainly caused by dermatophytes of the genus Trichophyton, but occasionally by nondermatophytic fungi including Aspergillus spp. (Moreno and Arenas, 2010). Aspergillus niger, an opportunistic filamentous fungus, is found to be associated with nail onychomycosis in both toenails and fingernail lesions (Kim et al., 2012). Terbinafine showed higher cure rates, when administered to treat onychomycosis, especially those caused by Aspergillus niger (Gianni and Romano, 2004). TH is an antifungal drug with most frequent adverse effects after oral use are gastrointestinal disturbances such and mild abdominal pain (Sweetman, 2009). Topical administration can avoid its adverse 
effects, but the limited solubility of $\mathrm{TH}$, makes its have a quite low bioavailability, especially in topical dosage forms.

Thus, the aim of the present study was to compare the skin permeation of $\mathrm{TH}$ as a model drug using different vesicular carriers, viz. transfersome, menthosome and ethosome to improve its antifungal activity. In this study, we adopt transfersome formulations with different ratios of nonionic surfactant (Span 80 and Tween 80 with HLB 4.5 and 15 respectively). First, the terbinafine transfersome formulations were prepared to obtain the optimized desirable formula which made a contribution to deformability and small particle size and high entrapment efficiency of drug obtained. The relationship between formulation factor and response variables was predicted, and the optimal formulation was also optimized using Design Expert@. Finally, comparative study of TH transfersome, menthosome and ethosome nanosize formulations via skin permeation and antifungal activity were studied.

\section{Materials and Methods}

\section{Materials}

Terbinafine hydrochloride was obtained as a gift from Memphes company, cairo, Egypt. Tween 80, Span 80, Chloroform and methanol were obtained from SigmaAldrich (St. Louis, MO). L- $\alpha$-phosphatidylcholine (soy 95\%) was purchased from Lipoid Phospholipid $\mathrm{GmbH}$, Germany. Cetremide was obtained from Winlab Chemicals; U.K. Ethyl alcohol was purchased from Algomhorya Company, Cairo, Egypt. Sodium hydroxide and potassium dihydrogen phosphate were purchased from ADWIC Company, Cairo, Egypt. All chemicals and reagents used were of analytical grade.

\section{Methodology}

\section{Development and Optimization of Transfersome Formulations}

Mixture Design was applied approach to identify the best proportion of each component in order to optimize multiple responses simultaneously. Effect of type and proportion of each component (nonionic surfactant) Span-80 ( $\left.\mathrm{X}_{1}\right)$ and Tween $80\left(\mathrm{X}_{2}\right)$ were studied. Mixture design containing two components was carried out to optimize transfersome formulations. The response in a mixture experiment usually is described by a polynomial function. This function represents how the components affect the response. The responses measured were particle size $\left(\mathrm{Y}_{1}\right)$ and entrapment efficiency $\left(\mathrm{Y}_{2}\right)$. To better study the shape of the response surface, the natural choice for a design would be the one whose points are spread evenly over the whole simplex. A simplex centroid design only includes the centroid points. A simplex centroid design can be used to fit the following model.

$$
\mathrm{Y}=\beta_{1} \mathrm{X}_{1}+\beta_{2} \mathrm{X}_{2}+\beta_{1,2} \mathrm{X}_{1} \mathrm{X}_{2} \quad \text { Eq. } 1
$$

The above model is called special cubic model. Note that the intercept term is not included due to the correlation between all the components (their sum is 100\%) (Cornell, 2011).

$\mathrm{Y}$ is the measured response associated with each factor-level combination, $\mathrm{X}_{1}$ and $X_{2}$ are the factors studied, $\beta_{1}, \beta_{2}$ and $\beta_{1,2}$ are the regression coefficients. The equation enables to study the effect of each factor and their interaction over the considered responses. Mixture design containing three components was carried out to 
optimize transfersome formulations (Nutan et al., 2007). Table I illustrates the independent variables and their level used in this study. This optimized formula was prepared and characterized to compare the observed values of $Y_{1}$ and $Y_{2}$ with the predicted responses. Preparation and determination of the vesicle size and EE were done. The optimized TH transfersome formulation was compared to $\mathrm{TH}$ of menthosome and ethosome formulations.

\section{Preparation of Terbinafine hydrochloride by different techniques (Transfersome, Menthosome and Ethosome)}

Transfersomes-loaded terbinafine hydrochloride were prepared using lipid film hydration technique previously described (Jain et al., 2003) with little modifications using combination of the edge activators (Span 80 and Tween 80). The drug (10mg), phosphatidylcholine $(850 \mathrm{mg})$ and edge activator $(150 \mathrm{mg})$ were mixed together and dissolved in an organic solvent mixture of chloroform and methanol $(1: 1 \mathrm{v} / \mathrm{v})$ then placed in a clean, dry round bottom flask. The organic solvent was removed by rotary evaporation under reduced pressure at $40^{\circ} \mathrm{C}$. The rotor speed was adjusted to $60 \mathrm{rpm}$. Complete removal of the organic solvent was accomplished after 120 minutes resulting in a homogenously distributed thin film on the wall of the flask. The deposited film was hydrated with a solution of phosphate buffer ( $\mathrm{pH} \mathrm{6.5)} \mathrm{by} \mathrm{rotation} \mathrm{for} 1 \mathrm{hr}$ at $45^{\circ} \mathrm{C}$. The vesicular suspension was then sonicated using probe sonicator at $4{ }^{0} \mathrm{C}$ for $15 \mathrm{~min}$ to reduce the size of the vesicles. The preparation of menthosome formula using cationic surface active agent (cetrimide) edge activators at the optimum concentration $10 \mathrm{mg}$ was prepared. The method for preparation of menthosome was nearly the same of the aforementioned method of transfersome formulations; just replace the edge activator nonionic by cationic surfactant (Duangjit et al., 2014). Ethosome was prepared using cold method. The drug (10mg) and phosopholipid $(850 \mathrm{mg})$ were dissolved in ethanol $(30 \%)$ in covered vessel at room temperature by vigorous stirring. Heat the mixture up to $40{ }^{\circ} \mathrm{C}$ in a water bath. In a separate vessel heat the water up to $40{ }^{\circ} \mathrm{C}$ and add to the above mixture slowly in a fine stream under constant stirring at $700 \mathrm{rpm}$, on completion of adding, continue stirring for another $5 \mathrm{~min}$ at $40{ }^{\circ} \mathrm{C}$ and cool the resultant vesicle suspension at room temperature. Ethosome was subjected to sonication at $4{ }^{0} \mathrm{C}$ using probe Sonicator for $1 \mathrm{~min}$ (Zhang et al., 2012).

\section{Vesicles characterization}

\section{Investigation of vesicles size, polydispersity index and zeta potential}

Average vesicles size, the corresponding Polydispersity index and the zeta potential were determined using Malvern Zetasizer (Zetasizer; Malvern Zetasizer, model Nano ZS, U.K). For size measurements, the vesicular suspension was mixed with PBS ( $\mathrm{pH}$ 6.5). Three measurements with 10 sub-runs were performed for each sample. The data of the size measurements were analyzed by the general purpose mode, and zeta potential measurements

were processed in the monomodal mode (Zhang et al., 2012).

\section{Entrapment Efficiency of TH formulations}

Indirect method was employed to calculate the amount of TH entrapped in each formula. Samples from the prepared formulae were transferred to eppendorff tubes and refrigerated at $-20^{\circ} \mathrm{C}$. The frozen samples were centrifuged at $14000 \mathrm{rpm}$ for 30 minutes. The supernatant solutions were assayed for drug content 
spectrophotometrically (U.V spectrophotometer; Schimadzu, model UV-1800, Tokyo, Japan) after dilution with phosphate buffer $\mathrm{pH}$ 6.5. The entrapment efficiency (EE) of TH was determined spectrophotometrically at $223 \mathrm{~nm}$. The TH content was calculated using the calibration curve. Each sample was analyzed in triplicate. The \% EE was calculated from the equation (Zhang et al., 2012).

$$
\% \mathrm{EE}=\frac{\text { Initial drug Concentration }- \text { Concentration of free drug }}{\text { Initial drug Concentration }} \times 100
$$

\section{Physicochemical study of TH nanovesicle.}

Comparative elasticity study of different T-optimize, menthosome and ethosome formulations were carried out by extrusion measurement through a locally fabricated stainless steel pressure filter holder. The vesicles were extruded through polycarbonate filter with $(19 \mathrm{~mm}, 0.1 \mu \mathrm{m})$ a pore size of 100 to $120 \mathrm{~nm}$ at a constant pressure of 0.17 $\mathrm{MPa}$. The elasticity was measured as a function of time (i.e. the time taken for the extrusion of $10 \mathrm{ml}$ Transfersomal suspension) (Cevc and Gebauer, 2003). The experiments were carried out in triplicates to obtain an average value.

Differential scanning calorimetry (DSC) was performed to investigate the thermal behavior of the drug and the freeze-dried drug-loaded transfersome, menthosome and ethosome formulations.

Fourier transforms infrared (FT-IR) of the drug alone and selected T-optimize, menthosome and ethosome formulations spectra studied in the FT-IR section were obtained in the range 4000-400 $\mathrm{cm}^{-1}$ by using (Perkin Elmer Spectrum One, Model 16 PC, Rheinstetten, Germany). Transparent discs were prepared by mixing each sample with $\mathrm{KBr}$. The mixture was compressed and tested.

The TEM pictures of T-optimize desirable formula, menthosome and ethosome nanosize formulae were obtained using TEM microscope (JEM-1230). Samples were negatively stained with a $1 \%$ aqueous solution of Phosphotungstic acid. Vesicle suspension samples were dried on a carbon coated grid for staining. After drying, the specimen was viewed under the microscope at 10-100 k-fold enlargements at an accelerating voltage of $100 \mathrm{kV}$ (Ahmed, 2015).

\section{Ex-vivo skin permeation study}

An accurately quantity amount of TH transfersome, menthosome and ethosome formulations suspension, equivalent to definite amount of $\mathrm{TH}$, were suspended in the Franz diffusion pool (DENG et al., 2011) having the diameter of $2 \mathrm{~cm}$ and diffusion area $3.14 \mathrm{~cm}^{2}$. The rat skin was used (All institutional and national guidelines for the care and use of laboratory animals were followed). The isolated skin of the rat with thickness of $0.5 \mathrm{~mm}$ was fitted between the supply pool and the reception pool and was placed in the transdermal diffusion apparatus (TK-20 A, Shanghai Kaikai Technology Trade Co., Ltd, China). The reception pool was fulfilled with $50 \mathrm{ml}$ phosphate buffer $\mathrm{pH} 6.5$ with constant stirring at a speed $(120 \mathrm{rpm})$ at $37 \pm 1{ }^{0} \mathrm{C}$. At predetermined time intervals $(0.25,0.5,1,2,4,6,8$ and $12 \mathrm{~h}), 1 \mathrm{~mL}$ of the reception liquid was withdrawn for analysis and replaced with equal volume of fresh reception solution at the same temperature to maintain a constant volume. After the last sample was collected, the concentration of the drug was determined spectrophotometrically at $223 \mathrm{~nm}$. All experiments were performed in triplicate with standard diviation $( \pm \mathrm{SD})$. Then the skin was taken off and washed and extract $\mathrm{TH}$ that remained in the skin. The supernatant 
liquid samples were collected and assayed to know the amount of drug remained on the skin. The release data of $\mathrm{TH}$ formulations from the prepared batches were fitted to different kinetic orders or models; zero order, first order, diffusion, and Korsmeyer model to explore the best fit order/model and the exact mechanism of drug release from the TH nano-vesicle size (El-Say, 2016).

\section{Stability studies}

Stability of the T-optimize, menthosome and ethosome were determined by assaying the vesicles size and entrapment efficiency at time $0,1,2$ and 3 months by the same aforementioned procedures. Three samples of each of the selected formulae were used and stored at $4{ }^{\circ} \mathrm{C}$ temperature (Zhang et al., 2012). All formulae were kept in tightly closed clean umber colored glass container (El Zaafarany et al., 2010). Size change rate (SCR) was calculated from the following equation(Shen et al., 2014)

$$
\mathrm{SCR}=\frac{\mathrm{S}_{3}-\mathrm{S}_{0}}{\mathrm{~S}_{0}} \times 100
$$

Where $S_{3}$ is the vesicle size after three months and $S_{0}$ is the initial vesicle size at the start of experiment. Change are not considered relevant if SCR value is less than 10\% (Manconi et al., 2016).

\section{In vitro antifungal assay of TH formulations}

Pure cultures of two test fungi, one yeast-like Candida albicans (ATCC 10231) and one filamentous Aspergillus niger (ATCC 16404), were used in this study. These fungi were cultured on Sabouraud dextrose agar. The antifungal activities of TH formulations were systematically performed test fungi by agar cup plate diffusion method based on the methodology used by bauer et al., (Bauer et al., 1966) with some modifications. The sterile Sabouraud dextrose agar was poured into sterile Petri plates aseptically and allowed to solidify at room temperature. Petri plates were flooded with $200 \mu 1$ of the fresh fungal suspension in sterilized saline equivalent to McFarland 0.5 standard solution $\left(1.5 \times 10^{8} \mathrm{CFU} / \mathrm{mL}\right)$ and uniformly distributed by sterile glass rod, then allowed to dry for 20 minutes with lid in place. In each plate, 3 wells of $6 \mathrm{~mm}$ diameter were made with a sterile borer, and the agar plugs were taken out carefully so as not disturb the surrounding medium. Precisely $100 \mu \mathrm{l}$ of the $\mathrm{TH}$ formulations (transfersome, menthosome and ethosome) were added to one cup aseptically and labeled accordingly as well as blank (composed of all components of formulation except $\mathrm{TH})$ and pure $\mathrm{TH}$ suspension. After holding the plates at room temperature for $1 \mathrm{~h}$ to allow diffusion of test samples into the agar, the plates were incubated at $28^{\circ} \mathrm{C}$ for 48 hours for fungi. The diameter of the clear zone of inhibition surrounding each well was measured twice at right angles and the average of the two readings was recorded to the nearest $\mathrm{mm}$. The inhibition effects of formulations were compared with that of blank and drug. The zone of inhibition above $7 \mathrm{~mm}$ in diameter was taken as positive result.

\section{Results and discussion}

Terbinafine Hydrochloride is lipophilic and could appropriately permeate through corneous layer into nail plate. Poor permeability of its external preparation leads to poor curative effect. TH is a good candidate for nano-vesicle forms (transfersomes, menthosome and ethosome) to enhance its bioavailability via 
transdermal route. This study is aimed to prepare high skin penetration $\mathrm{TH}$ vesicle nanosize with high entrapment efficiency (EE) and good stability. The influence of various edge activator (Span and Tween) formulations and processing parameters that affect the vesicle size and EE were investigated. The prepared TH nano-vesicle forms were utilized a simplex centroid design to obtain optimize desirable formula using suitable tool (Statgraphics_plus software) to identify the parameters that are useful for this study.

Table 1 TH transfersomes independent and dependent variables used in the mixture simplex centroid design.

\begin{tabular}{llcr}
\hline & & \multicolumn{2}{c}{ Level } \\
\cline { 3 - 4 } & & Low & High \\
\hline Independent variables & $\mathrm{X}_{1}$ & 0 & 150 \\
Span 80 & $\mathrm{X}_{2}$ & 0 & 150 \\
Tween 80 & & Goal of optimization \\
Dependent variables & $\mathrm{Y}_{1}$ & \multicolumn{2}{c}{ Minimize } \\
Vesicle size & $\mathrm{Y}_{2}$ & \multicolumn{2}{c}{ Maximize } \\
Entrapmentefficiency & \multicolumn{3}{c}{} \\
\end{tabular}

Table 1, illustrates the lower and higher level for the independent studied variables of different nanionic surfactant (Span $\mathrm{X}_{1}$ and Tween $\mathrm{X}_{2}$ ). The mixtures with different concentration of edge activator (EA) having different HLB values were selected to enhance the drug entrapment efficiency and elasticity of the vesicles size.

The composition of the formulations obtained by the software mixture simplex centroid design showed in Table 2.

Table 2 Composition of mixture simplex centroid design used for $\mathrm{TH}$ transfersome formulations and comparative nano-vasicle formulations.

\begin{tabular}{ccccccc}
\hline Formulae & Drug & Phospholipid & Span 80 & Tween 80 & Cetrimide & Ethanol \\
\hline F $_{1}$ & 10.0 & 850 & 150.0 & 0.000 & - & - \\
F $_{2}$ & 10.0 & 850 & 75.00 & 75.00 & - & - \\
F $_{3}$ & 10.0 & 850 & 0.000 & 150.0 & - & - \\
F $_{4}$ & 10.0 & 850 & 75.00 & 75.00 & - & - \\
F $_{5}$ & 10.0 & 850 & 112.5 & 37.50 & - & - \\
F $_{6}$ & 10.0 & 850 & 37.50 & 112.5 & - & - \\
\hline ComparativeNano-vesicle Formulations & & & - \\
\hline T- optimize & 10.0 & 850 & 59.10 & 90.90 & - & - \\
Menthosome & 10.0 & 850 & - & - & 10.0 & - \\
Ethosome & 10.0 & 850 & - & - & - & 35 \\
\hline
\end{tabular}


In this study, the effect of the formulation parameters affecting the preparation of TH transfersomes was studied using mixture optimization design. This work is the first study in the development of optimized $\mathrm{TH}$ transfersome preparation suitable for transdermal drug delivery. The transfersome formulations were prepared at ratio PL:EA $85: 15$ as the fixed ratios in all formulations. The edge activator nonionic surfactants with different HLB values (Span and Tween 4.5 and 15 HLB respectively) were studied and develop the effect of nonionic surfactant on transfersome formulations. The desirable and optimized formula via vesicle size and entrapment efficiency was obtained.

\section{Characterization of the vesicle size, zeta potential, EE and deformability of lipid vesicle}

Transfersome-proposed formulations were characterized via vesicle size, size distribution, zeta potential, EE and deformability of the vesicles. TH is a good choice drug for encapsulation into nano-vesicle forms (transfersomes, menthosome and ethosome) to enhance its permeability power via transdermal route. The results of transfersome formulations were shown in Table 3 . The vesicle size ranged from $(79.8$ to155 nm). Transfersome formulations containing Tween $80\left(\mathrm{~F}_{3}\right)$ and Tween combination with different ratios of Span $80\left(\mathrm{~F}_{2}, \mathrm{~F}_{4}, \mathrm{~F}_{5}\right.$ and $\left.\mathrm{F}_{6}\right)$ showed smaller vesicle size than those containing Span alone $\left(\mathrm{F}_{1}\right)$. Transfersomes showed narrow particle size distribution as indicated by low Polydispersity index. The zeta potential is the electric potential of the vesicle, including its ionic atmosphere. The zeta potential of transfersome formulations were obtained between $(-4.42$ to $-9.30 \mathrm{mV})$. The results of entrapment efficiency of TH transfersomes containing different kinds of surfactants are above $85 \%$. By comparing the optimum deformability ratio between lipid and edge activator $(85: 15 \%, w / w)$, it was found that, Tween only $\left(F_{3}\right)$ showed the highest deformability $(11.7 \mathrm{~s})$, the deformability of Tween combination with different ratios with Span $80\left(\mathrm{~F}_{2}, \mathrm{~F}_{4}, \mathrm{~F}_{5}\right.$ and $\left.\mathrm{F}_{6}\right)$ were smaller than that of transfersomes containing span alone $48.5 \mathrm{~s}\left(\mathrm{~F}_{1}\right)$. This could be attributed to the highly flexible and non-bulky hydrocarbon chains of Tween. Span had lower deformability than Tween due to their steroid-like structures which are bulkier than the hydrocarbon chains of Tween (El Zaafarany et al., 2010)

Table 3 Characterization the vesicle of TH transfersome formulations and comparative of three lipid vesicle types

\begin{tabular}{cccccc}
\hline Formulae & $\begin{array}{c}\text { Vesicle } \\
\text { Size } \\
(\mathbf{n m})\end{array}$ & $\begin{array}{c}\text { Zeta } \\
\text { Potential } \\
(\mathbf{m V})\end{array}$ & $\begin{array}{c}\text { Polydispersity } \\
\text { Index }\end{array}$ & $\begin{array}{c}\text { Entrapment } \\
\text { Efficiency } \\
(\%)\end{array}$ & $\begin{array}{c}\text { Deformability } \\
\text { value(sec) }\end{array}$ \\
\hline F $_{1}$ & $155.0 \pm 6.2$ & $-4.42 \pm 0.2$ & $0.322 \pm 0.05$ & $87.3 \pm 2.9$ & $48.4 \pm 2.6$ \\
F $_{2}$ & $85.60 \pm 5.4$ & $-9.30 \pm 0.4$ & $0.240 \pm 0.03$ & $92.2 \pm 3.7$ & $22.5 \pm 2.8$ \\
F $_{3}$ & $79.80 \pm 4.6$ & $-5.12 \pm 0.3$ & $0.263 \pm 0.02$ & $82.8 \pm 4.6$ & $11.7 \pm 1.8$ \\
F $_{4}$ & $84.50 \pm 5.7$ & $-8.50 \pm 0.5$ & $0.280 \pm 0.03$ & $91.4 \pm 6.4$ & $23.8 \pm 1.9$ \\
F $_{5}$ & $100.0 \pm 4.4$ & $-5.35 \pm 0.4$ & $0.292 \pm 0.04$ & $90.5 \pm 4.3$ & $35.6 \pm 3.2$ \\
\hline F & $83.42 \pm 3.8$ & $-7.88 \pm 0.6$ & $0.284 \pm 0.05$ & $85.6 \pm 5.2$ & $15.5 \pm 1.7$ \\
\hline ComparativeNano-vesicle Formulations & & & \\
\hline T-optimize & $80.40 \pm 4.6$ & $-8.28 \pm 0.7$ & $0.220 \pm 0.05$ & $93.54 \pm 5.3$ & $18.5 \pm 2.0$ \\
\hline Menthosome & $122.8 \pm 7.2$ & $8.77 \pm 0.8$ & $0.303 \pm 0.04$ & $93.86 \pm 6.8$ & $20.5 \pm 2.6$ \\
Ethosome & $67.80 \pm 5.4$ & $11.3 \pm 0.5$ & $0.248 \pm 0.05$ & $95.75 \pm 6.5$ & $13.9 \pm 1.5$ \\
\hline
\end{tabular}




\section{Parameters studied effect on the vesicle size and entrapment efficiency}

The effect of the studied variables on the vesicle size was investigated for TH transfersome. To illustrate the effect of the studied variables on the vesicle size when two variables are changing, estimated response surface and contours of estimated response surface (Figure1: A1 and A2) were constructed. The equation of the fitted model is:

$$
\mathrm{Y}_{1}=150.57 * \mathrm{X}_{1}+83.78 * \mathrm{X}_{2}-131.15 * \mathrm{X}_{1} \mathrm{X}_{2}
$$

The above equations were derived by the best-fit method to describe the main effect of process variables $\left(\mathrm{X}_{1}\right.$ and $\left.\mathrm{X}_{2}\right)$ and their interaction $\left(\mathrm{X}_{1} \mathrm{X}_{2}\right)$ on the responses $\mathrm{Y}_{1}$. Generally, a positive sign reflects a synergistic effect while a negative sign stands for an antagonistic effect. Analysis of variance (ANOVA) was done for the effect of each factor. One can conclude from the regression Equations and has the most significant synergistic effect ( $\mathrm{p}$-value $<0.05$ ) on vesicle size $\left(\mathrm{Y}_{1}\right)$. The results also revealed that the interaction effect between $\mathrm{X}_{1} \mathrm{X}_{2}$ has an antagonistic effect on vesicle size.

Both surfactants Span $\left(\mathrm{X}_{1}\right)$ and Tween $\left(\mathrm{X}_{2}\right)$ affect significantly on the vesicle size of the prepared transfersome at p-values of 0.0087 and 0.0091 respectively, which was also demonstrated in the Estimated Response Surface and contours Estimated Response Surface in (Figure 1: A1 and A2). As the surfactant HLB increased, the vesicle size decreased, which could be related to the affinity of the phospholipid used toward surfactant. The affinity was highest with Span followed by Tween. New finding were reported for the effect of different surfactant combinations on vesicle size and deformability of TH transfersomes. The vascular size was decreased and the elasticity of nanovesicle was enhanced by increasing Tween concentration with low concentration of Span $\left(\mathrm{F}_{6}\right)$ combination.

To study the effect of the variables on Entrapment efficiency when two variables are changing estimated response surface (Figure1: B1) and contours of estimated response surface (Figure1: B2) were investigated. The equation of the fitted model is: $\mathrm{Y}_{2}=87.524 * \mathrm{X}_{1}+82.02 * \mathrm{X}_{2}+24.36 * \mathrm{X}_{1} \mathrm{X}_{2}$
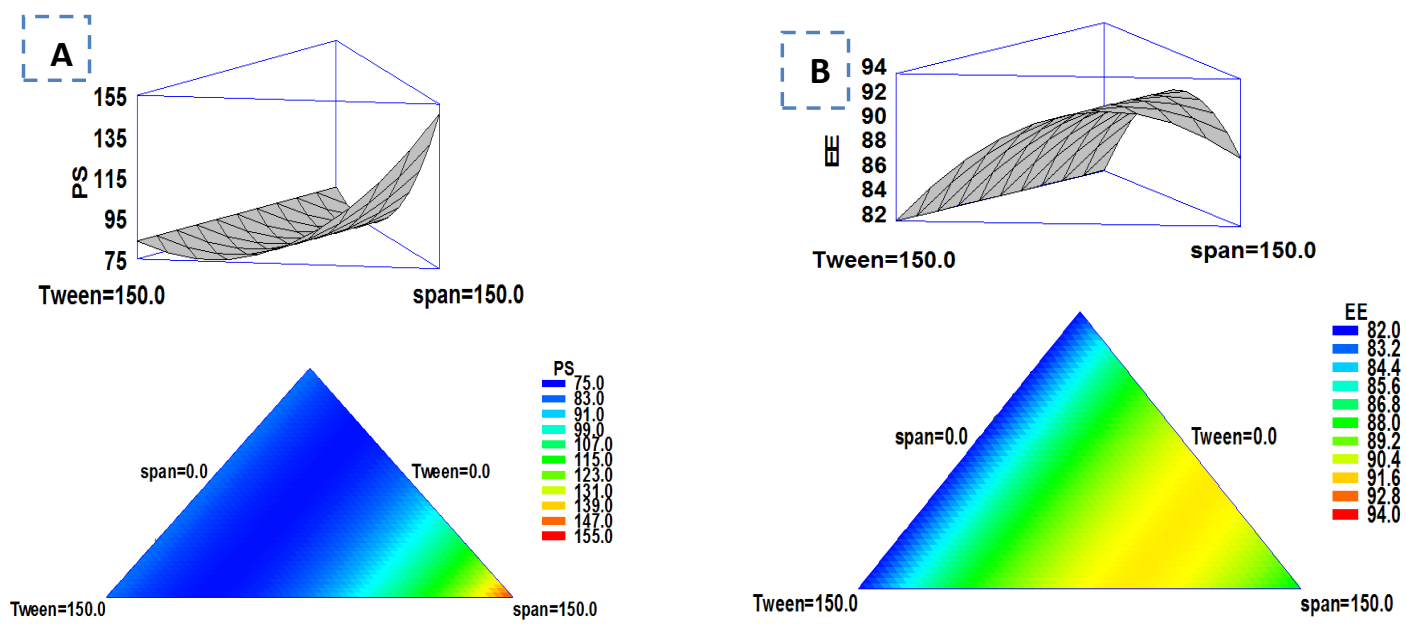
Fig. 1 Response surface plot estimating and contour estimated response the effect of span $\left(\mathrm{X}_{1}\right)$, and Tween $\left(\mathrm{X}_{2}\right)$ on the particle size $\left(\mathrm{Y}_{1}\right.$ represented in $\left.\mathrm{A}\right)$ and Entrapment efficiency $\left(\mathrm{Y}_{2}\right.$ represented in $\left.\mathrm{B}\right)$ of $\mathrm{TH}$ transfersome formulations.

From equations were derived by the best-fit method to describe the main effect of process variables $\left(\mathrm{X}_{1}\right.$ and $\left.\mathrm{X}_{2}\right)$ and their interaction $\left(\mathrm{X}_{1} \mathrm{X}_{2}\right)$ on the responses $\mathrm{Y} 2$. One can conclude from the regression Equations and Figures1; B1 and B2 has the most significant synergistic effect ( $\mathrm{p}$-value $<0.05$ ) on entrapment efficient $\left(\mathrm{Y}_{2}\right)$. The results also revealed that the interaction effect between $X_{1} X_{2}$ has a synergistic effect on $Y_{2}$. The combination of two surfactants having different HLB values producing high entrapment efficiency of drug loaded transfersome formulations in comparison to each surfactant alone (Jacob and Kr, 2013). In addition, HLB values of the surfactant affect the entrapment efficiency of the drug loaded nano-vesicle. Since, increasing HLB values decrease the entrapment efficiency of the drug. This finding was in agreement with previous studies demonstrated the effect of surfactant HLB on the entrapment of drugloaded transfersomes (Jacob and Kr, 2013).

From the composition of the multiple responses the optimized desirable formula was identified. The optimized formula was proposed to contain 59.10 and $90.90 \%$ of $\mathrm{X}_{1}$ and $\mathrm{X}_{2}$, respectively. This optimized formula was prepared and characterized for its vesicle size and EE. The predicted values obtained from optimization were compared to the observed ones in which the residual was calculated and presented in Table 4. The optimized formula where further characterized and compared with other nano-vesicle system (menthosome and ethosome).

Table 4 Multiple response optimization of Terbinafine transfersome

\begin{tabular}{lcccc}
\hline Factor & Low level & High level & Optimum level \\
\hline $\mathrm{X}_{1}$ & & 0 & 150 & 59.10 \\
$\mathrm{X}_{2}$ & & 0 & 150 & 90.90 \\
\hline Response & & Predicted & Observed & Residual \\
\hline Vesicle size(nm) & $\mathrm{Y}_{1}$ & 78.75 & 80.40 & 1.65 \\
Entrapmentefficiency & $\mathrm{Y}_{2}$ & 92.67 & 93.54 & 0.87 \\
\hline \hline
\end{tabular}

Comparative studies between T-optimize, menthosome and ethosome via vesicle size, zeta potential, EE and deformability of lipid vesicle

The result of T-optimize, menthosome and ethosome formulations were shown in Table 3. The vesicle size was $80.40,122.80$ and $67.80 \mathrm{~nm}$ respectively. The vesicle size of ethosome and T-optimize were smaller than the menthosome formula. In particular, the ethosomal formulation had a much smaller particle size. This may be attributed to the high concentration of ethanol, which could cause a reduction in vesicle size. In addition, the zeta potential of T-optimize, menthosome and ethosome was -8.28 , 8.77 and 11.30 respectively. The charge of zeta potential was depending on the types of surfactant used in T-optimize and menthosome while in ethosome attributed to the drug. 
The results of entrapment efficiency of $\mathrm{TH}$ nanosize formulation were ranged from 93.54 to 95.75. Finally, the deformability values of TH nano-vesicle were showed of Toptimize $18.5 \mathrm{sec}$, menthosome $20.50 \mathrm{sec}$ and ethosome $13.9 \mathrm{sec}$. This could be attributed to the vesicle size of TH nanovasicle formulation (Touitou et al., 2000; Zhang et al., 2012). The EE of three formulations (T-optimize, menthosome and ethosome) of lipid vesicles were high. These may be contributed to the weight ratio of PL to surfactant in combination of T-optimize formula. While, the greater distribution of TH loaded in menthosome core was attributed to lipid bilayer containing cetrimide. The ethosome vesicles containing high ethanol concentrations that had high solubility of $\mathrm{TH}$, corresponding to the formation of a phase with interpenetrating hydrocarbon chains, which contributed to improving the EE of the vesicles (Jain et al., 2007).

\section{Physicochemical characterization of TH nano-vesicle formulations}

The thermal characteristic using DSC of the TH and comparative (T-optimize, menthosome and ethosome) formulations were studied. DSC is a tool used to investigate the crystalline or amorphous nature of the drug within the developed formulations and to elucidate any possible interactions with other ingredients. TH pure drug thermogram showed characteristic thermal peak at reported melting point $\left(198^{\circ} \mathrm{C}\right)$ (Kuminek et al., 2013). Thermograms for vesicular systems (T-optimize, menthosome and ethosome) showed disappearance of TH thermal peak which indicates entrapment of the drug into the vesicles and loss of its crystal properties. In addition, thermograms of vesicle systems showed new endothermic peaks which the most probably due to the transition temperature (Tm) of PL.

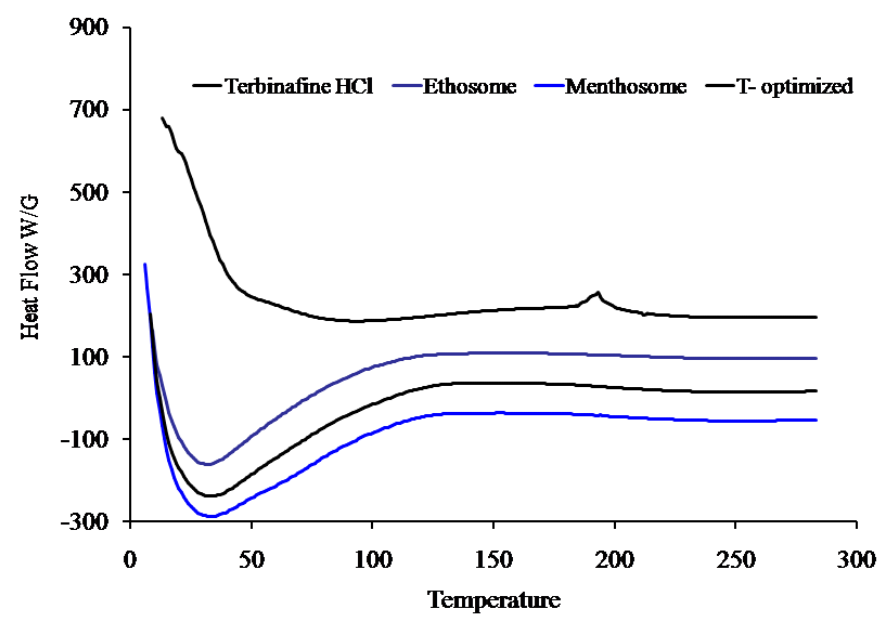

Fig. 2 DSC thermogram of Terbinafine HCL and drug-loaded transfersome, menthosome and ethosome formulations.

From figure 3, the FTIR spectrum of pure TH showed $\mathrm{C} \equiv \mathrm{C}$ stretching bands at $2443.79 \mathrm{~cm}^{-1}$, aromatic $\mathrm{C}=\mathrm{C}$ stretching bands at $1521 \mathrm{~cm}^{-1}$, aromatic $\mathrm{C}-\mathrm{H}$ stretching bands at $3041.08 \mathrm{~cm}^{-1}$, and $\mathrm{C}-\mathrm{N}$ bands at 1132.49 and aliphatic $\mathrm{C}-\mathrm{H}$ stretching bands at $2969 \mathrm{~cm}^{-1}$. (Pohle et al., 2001). The lyophilized nano-vesicle TH formulation FTIR spectrum showed slightly change of some bands. Presence of Phospholipid and surfactants used did not produce any major shift in the principal peaks of HT and also the presence of one ingredient did not produce shift in the peaks of other ingredients. This indicates that there was no interaction between drug and the Additives used in the study. Hence FTIR spectral analysis proved the compatibility of the drug and additives 
used (Kumar, 2012). The above finding confirms compatibility of the formulation ingredients.

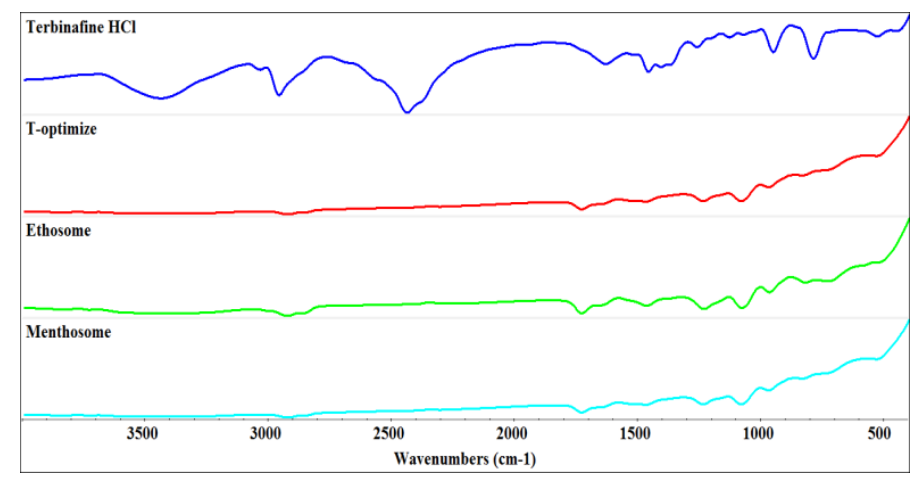

Fig. 3 IR spectra of Terbinafine $\mathrm{HCl}$ and drug-loaded transfersome, menthosome and ethosome formulations.

\section{Vesicle shape characterization of TH nano-vesicle formulations}

Investigation of morphology for three formulations (transfersome, menthosome and ethosome) using TEM revealed that all types were spherical shape with a few difference in edge of the vesicle surface which attributed to different types of edge activator used (Figure 4).

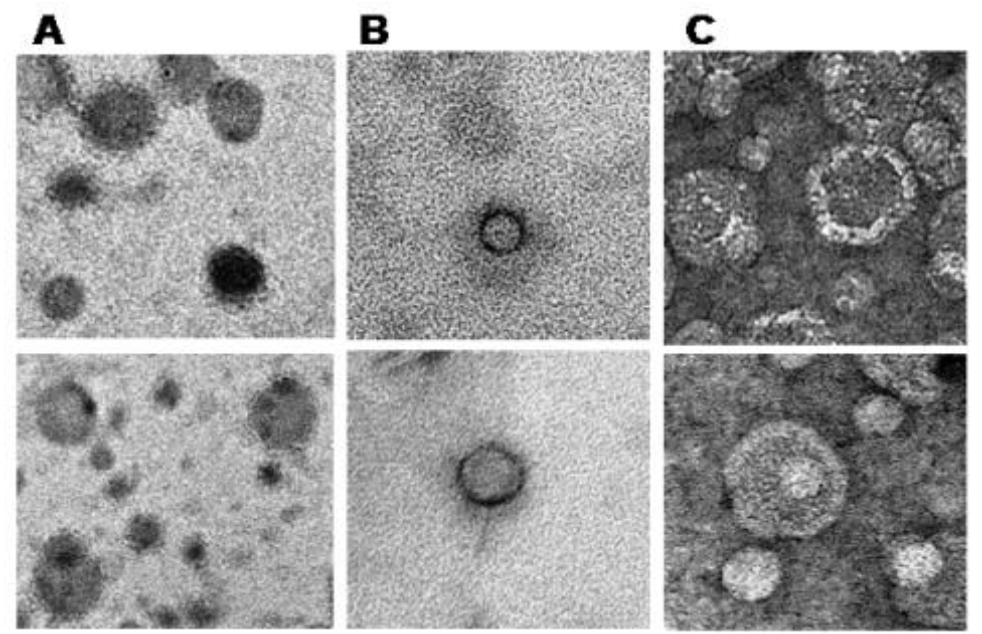

Fig. 4 TEM pictures of Terbinafine loaded transfersome (A), menthosome (B) and ethosome (C) formulations.

\section{Ex vivo skin permeation study of TH nano-vesicle formulations}

The Ex vivo permeation of $\mathrm{TH}$ loaded lipid vesicles were carried out for different formulations (T-optimize, menthosome and ethosome) and drug suspension. The Ex vivo permeation study of the T-optimize, menthosome, and ethosome showed a considerable difference, more than 3,3.5 and 4-folds enhancement release respectively when compared with the drug suspension as showed in Figure 5. These nano-vesicle formulations had a marked impact on the ex vivo release of $\mathrm{TH}$ in phosphate buffer $\mathrm{pH}$ 5.5. TH ethosome nano-size led to a considerably faster release than transfersome and menthosome formulations. In ethosomes, ethanol is a well-known permeation enhancer. The effect of ethanol on vesicle fluidity as well as a dynamic interaction between 
ethosomes and the stratum corneum, may contribute to the delivery properties of ethosomes (Dayan and Touitou, 2000). These reasons allow ethosomes to permeate more easily and deeper into skin. While, TH transfersome formulation (T-optimize) was accumulate in skin. Thus, TH release from the vesicles in the stratum corneum is an important step that will affect transdermal flux. In addition, the zeta potential of transfersomes is more negative. This might explain why transfersomes can't effectively penetrate through the skin because the skin has a negative charge (Jain et al., 2007). Finally, the poor drug release resulted in retention of the drug within vesicles in the stratum corneum and elastic vesicles served as a slow release depot system (HoneywellNguyen and Bouwstra, 2003). These results are consistent with a previously illustrated by many researchers (Gillet et al., 2011; Yang et al., 2015; Zhang et al., 2012). In general, the mechanism of drug release from the prepared transfersome could be attributed to a diffusion control mechanism as previous investigation (Hathout et al., 2007; Nasr et al., 2008) data not showed.

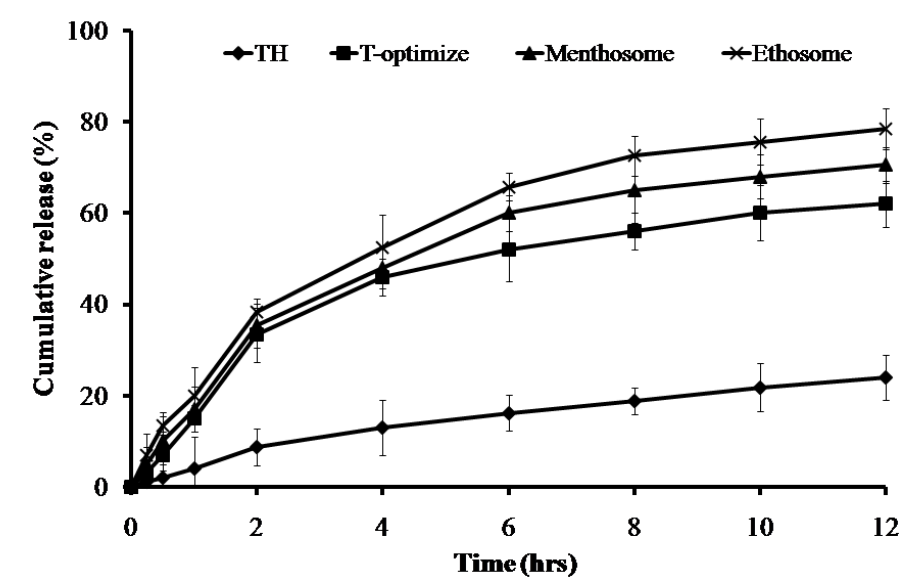

Fig. 5 Ex vivo Comparison between the release profiles of different T-optimize, menthosome and ethosome formulations and $\mathrm{TH}$ suspension via $12 \mathrm{~h}$ by Mice Skin, in phosphate buffer $\mathrm{pH} 5.5$.

\section{Stability of TH lipid vesicles}

In Figure $6 \mathrm{~A}$ and $\mathrm{B}$, the nano-vesicle formulations were determined at 0, 1, 2, and 3 months following vesicle preparation. The vesicle size and entrapment efficiency of the three lipid vesicle form showed no significant changes within 3 months at $4{ }^{\circ} \mathrm{C}$ (El Zaafarany et al., 2010; Naik, 2013). Size change rate (SCR) was also calculated for each vesicle system. SCR less than $10 \%$ is reported to be acceptable (Manconi et al., 2012). After three months, TH of nano-vesicle formulations showed SCR of Toptimize, menthosome and ethosome 9.20, 3.25 and 14.7\% respectively. Ethosome was reported to be stable at refrigeration temperatures (Chandel et al., 2012). However, this may be due to PL slightly aggregations upon storage at low temperature (Lee and Hong, 2014). This result of drug retained shows that the three lipid vesicle systems are stable under the given conditions. 

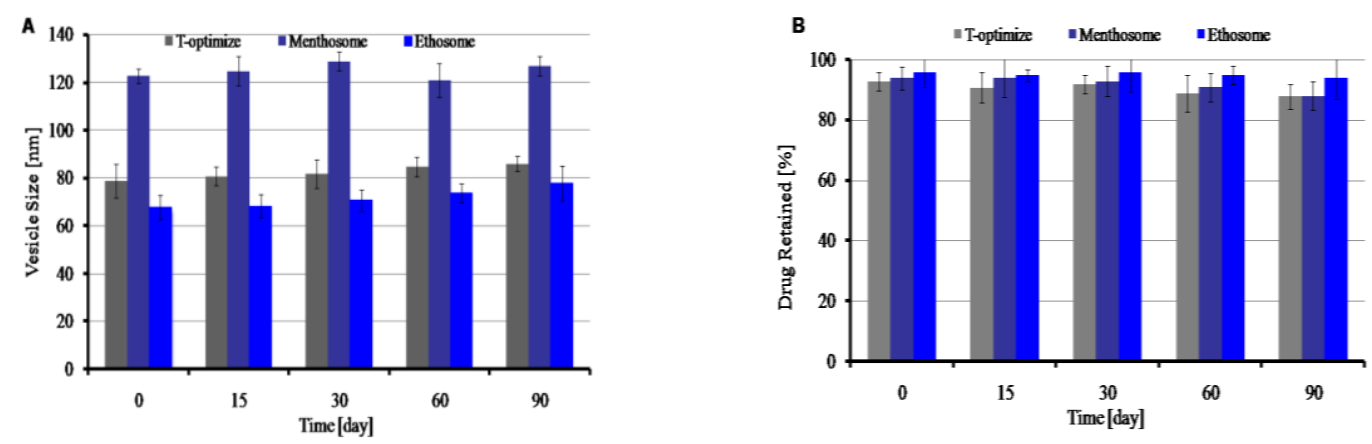

Fig. 6 Effect of storage stability on the vesicle size (A) and drug retained (B) of Toptimize, menthosome and ethosome formulations.

\section{In vitro antifungal activity of $\mathrm{TH}$ nanovesicular size formulations}

The antifungus activity of the three formulations and drug showed good antifungal activity against the filamentous Aspergillus niger. However, there was no activity against $C$. albicans. Significantly, the size of zones of inhibition indicated increasing in the antifungal activity of TH transfersome and $\mathrm{TH}$ menthosome; however, there was some inhibition of growth in case of TH ethosome formula when compared with drug alone (Table 5 and Figure 7).

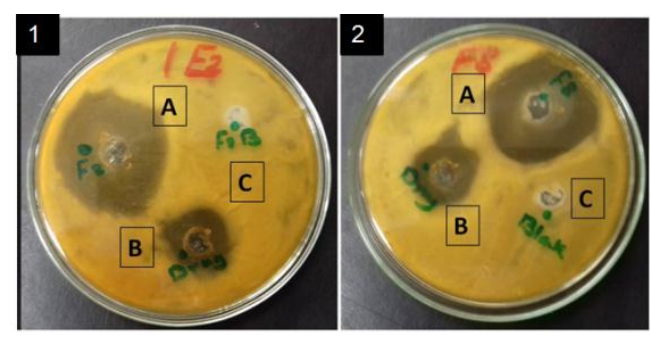

Fig.7 The antifungal activities of prepared formulations of drug loaded T-optimize (1) and Menthosome (2), against A. niger in comparison to blank and drug alone. (Formula A; Drug B; Blank C).

Table 5 Antifungal activity profile of drug and three tested formulations by agar well diffusion method

\begin{tabular}{l|c|cc|cc|cc}
\hline \multirow{2}{*}{ Fungus } & \multicolumn{6}{|c}{ Inhibition Zone } \\
\cline { 2 - 8 } & TH & \multicolumn{2}{|c|}{ T-optimize } & \multicolumn{2}{|c}{ Menthosome } & \multicolumn{2}{c}{ Ethosome } \\
\cline { 2 - 8 } & Drug & Blank & Test & Blank & Test & Blank & Test \\
\hline A. niger & 15 & - & 29 & - & 28 & 10 & 30 \\
C. albicans & - & - & - & - & - & - & - \\
\hline
\end{tabular}

\section{Conclusion}

From the aforementioned study, we can used the optimization technique with different formulation and processing variables to obtain optimize desirable formula with optimum combination ratio of Span $(59.10 \%)$ and Tween $(90.90 \%)$ with small vesicle size $(80.40 \mathrm{~nm})$ and high EE $(93.54 \%)$. The optimum formula was characterized by flexible lipid vesicles which can respond to rapid shape deformation, which enables 
them to pass through skin pores and more than 3-folds higher in vitro permeation when compared with the drug suspension. The accumulation of the TH transfersomes in the skin is high with low permeation power rather than menthosome and ethosome. Ethosome improve drug delivery with greater improvement in skin permeation than improvement in skin deposition. Transfersomes have the advantage that high concentrations of drugs can be localized at the site of action, where the goal is to reduce the systemic adsorption and minimize systemic side effects.

\section{Conflict of interest}

The authors report no conflict of interest in this work.

\section{REFERENCES}

Ahmed, T. A. (2015). Preparation of transfersomes encapsulating sildenafil aimed for transdermal drug delivery: Plackett-Burman design and characterization. Journal of liposome research 25, 1-10.

Bauer, A., Kirby, W., Sherris, J. C., and Turck, M. (1966). Antibiotic susceptibility testing by a standardized single disk method. American journal of clinical pathology 45, 493.

Cevc, G. (2003). Transdermal drug delivery of insulin with ultradeformable carriers. Clinical pharmacokinetics 42, 461-474.

Cevc, G., and Blume, G. (1992). Lipid vesicles penetrate into intact skin owing to the transdermal osmotic gradients and hydration force. Biochimica et Biophysica Acta (BBA)-Biomembranes 1104, 226-232.

Cevc, G., Blume, G., and Schätzlein, A. (1997). Transfersomes-mediated transepidermal delivery improves the regio-specificity and biological activity of corticosteroids in vivo. Journal of controlled release 45, 211-226.

Cevc, G., and Gebauer, D. (2003). Hydration-driven transport of deformable lipid vesicles through fine pores and the skin barrier. Biophysical journal 84, 10101024.

Cevc, G., Schätzlein, A., and Blume, G. (1995). Transdermal drug carriers: basic properties, optimization and transfer efficiency in the case of epicutaneously applied peptides. Journal of Controlled Release 36, 3-16.

Chandel, A., Patil, V., Goyal, R., Dhamija, H., and Parashar, B. (2012). Ethosomes: A novel approach towards transdermal drug delivery. J Pharm Chem Sci 1, 563-569.

Cornell, J. A. (2011). Experiments with mixtures: designs, models, and the analysis of mixture data, Vol 895: John Wiley \& Sons).

Dayan, N., and Touitou, E. (2000). Carriers for skin delivery of trihexyphenidyl $\mathrm{HCl}$ : ethosomes vs. liposomes. Biomaterials 21, 1879-1885.

DENG, H., ZHANG, S., LIN, H.-q., ZHANG, X.-l., BU, S., and LI, X.-w. (2011). Study of Transdermal Diffusion Experiment of 2 Kinds of Terbinafine Hydrochloride Cream in Vitro [J]. China Pharmacy 29, 018. 
Dragicevic-Curic, N., Gräfe, S., Gitter, B., Winter, S., and Fahr, A. (2010). Surface charged temoporfin-loaded flexible vesicles: in vitro skin penetration studies and stability. International journal of pharmaceutics 384, 100-108.

Duangjit, S., Obata, Y., Sano, H., Kikuchi, S., Onuki, Y., Opanasopit, P., Ngawhirunpat, T., Maitani, Y., and Takayama, K. (2012). Menthosomes, novel ultradeformable vesicles for transdermal drug delivery: optimization and characterization. Biological and Pharmaceutical Bulletin 35, 1720-1728.

Duangjit, S., Obata, Y., Sano, H., Onuki, Y., Opanasopit, P., Ngawhirunpat, T., Miyoshi, T., Kato, S., and Takayama, K. (2014). Comparative study of novel ultradeformable liposomes: menthosomes, transfersomes and liposomes for enhancing skin permeation of meloxicam. Biological and Pharmaceutical Bulletin 37, 239-247.

El-Say, K. (2016). Optimized gastroretentive floating carvedilol tablets: an approach for prolonged gastric residence time and enhanced absorption. Journal of Applied Pharmaceutical Science Vol 6, 012-019.

El Maghraby, G. M., Barry, B. W., and Williams, A. C. (2008). Liposomes and skin: from drug delivery to model membranes. european journal of pharmaceutical sciences 34, 203-222.

El Zaafarany, G. M., Awad, G. A., Holayel, S. M., and Mortada, N. D. (2010). Role of edge activators and surface charge in developing ultradeformable vesicles with enhanced skin delivery. International Journal of Pharmaceutics 397, 164172.

Essa, E. A., Bonner, M. C., and Barry, B. W. (2004). Electrically assisted skin delivery of liposomal estradiol; phospholipid as damage retardant. Journal of controlled release $95,535-546$.

Gianni, C., and Romano, C. (2004). Clinical and histological aspects of toenail onychomycosis caused by Aspergillus spp.: 34 cases treated with weekly intermittent terbinafine. Dermatology 209, 104-110.

Gillet, A., Lecomte, F., Hubert, P., Ducat, E., Evrard, B., and Piel, G. (2011). Skin penetration behaviour of liposomes as a function of their composition. European Journal of Pharmaceutics and Biopharmaceutics 79, 43-53.

Hathout, R. M., Mansour, S., Mortada, N. D., and Guinedi, A. S. (2007). Liposomes as an ocular delivery system for acetazolamide: in vitro and in vivo studies. Aaps Pharmscitech 8, E1-E12.

Hiruta, Y., Hattori, Y., Kawano, K., Obata, Y., and Maitani, Y. (2006). Novel ultradeformable vesicles entrapped with bleomycin and enhanced to penetrate rat skin. Journal of controlled release 113, 146-154.

Honeywell-Nguyen, P. L., and Bouwstra, J. A. (2003). The in vitro transport of pergolide from surfactant-based elastic vesicles through human skin: a suggested mechanism of action. Journal of Controlled Release 86, 145-156.

Jacob, L., and Kr, A. (2013). A review on surfactants as edge activators in ultradeformable vesicles for enhanced skin delivery. 
Jain, S., Jain, P., Umamaheshwari, R., and Jain, N. (2003). Transfersomes-a novel vesicular carrier for enhanced transdermal delivery: development, characterization, and performance evaluation. Drug development and industrial pharmacy 29, 1013-1026.

Jain, S., Tiwary, A. K., Sapra, B., and Jain, N. K. (2007). Formulation and evaluation of ethosomes for transdermal delivery of lamivudine. Aaps Pharmscitech 8, 249-257.

Kim, D. M., Suh, M. K., Ha, G. Y., and Sohng, S. H. (2012). Fingernail onychomycosis due to Aspergillus niger. Annals of dermatology 24, 459-463.

Kumar, A., Pathak, K., and Bali, V. (2012). Ultra-adaptable nanovesicular systems: a carrier for systemic delivery of therapeutic agents. Drug discovery today 17, 1233-1241.

Kumar, J. R. (2012). Formulation and in-vitro Evaluation of Terbinafine HCL Transdermal Patches.

Kuminek, G., Rauber, G. S., Riekes, M. K., de Campos, C. E. M., Monti, G. A., Bortoluzzi, A. J., Cuffini, S. L., and Cardoso, S. G. (2013). Single crystal structure, solid state characterization and dissolution rate of terbinafine hydrochloride. Journal of pharmaceutical and biomedical analysis 78, 105-111.

Lee, C., Wei, X., Kysar, J. W., and Hone, J. (2008). Measurement of the elastic properties and intrinsic strength of monolayer graphene. Science 321, 385-388.

Lee, M., and Hong, M. (2014). Cryoprotection of lipid membranes for high-resolution solid-state NMR studies of membrane peptides and proteins at low temperature. Journal of biomolecular NMR 59, 263-277.

Mahale, N., Thakkar, P., Mali, R., Walunj, D., and Chaudhari, S. (2012). Niosomes: novel sustained release nonionic stable vesicular systems-an overview. Advances in colloid and interface science 183, 46-54.

Malakar, J., Sen, S. O., Nayak, A. K., and Sen, K. K. (2012). Formulation, optimization and evaluation of transferosomal gel for transdermal insulin delivery. Saudi pharmaceutical journal 20,355-363.

Manconi, M., Caddeo, C., Sinico, C., Valenti, D., Mostallino, M., Lampis, S., Monduzzi, M., and Fadda, A. (2012). Penetration enhancer-containing vesicles: composition dependence of structural features and skin penetration ability. European Journal of Pharmaceutics and Biopharmaceutics 82, 352-359.

Manconi, M., Sinico, C., and Fadda, A. M. (2016). Penetration Enhancer-Containing Vesicles for Cutaneous Drug Delivery. In Percutaneous Penetration Enhancers Chemical Methods in Penetration Enhancement, (Springer), pp. 93-110.

Mezei, M., and Gulasekharam, V . (1980). Liposomes-a selective drug delivery system for the topical route of administration I. Lotion dosage form. Life Sciences 26, 1473-1477.

Moreno, G., and Arenas, R. (2010). Other fungi causing onychomycosis. Clinics in dermatology 28, 160-163. 
Naik, U. S. (2013) The synthesis and characterisation of novel ultra-flexible lipidic vesicles using propanol, Department of Pharmacy and Biomedical Sciences, University of Central Lancashire, Preston, UK.

Nasr, M., Mansour, S., Mortada, N. D., and Elshamy, A. (2008). Vesicular aceclofenac systems: a comparative study between liposomes and niosomes. Journal of Microencapsulation 25, 499-512.

Nutan, M. T., Vaithiyalingam, S. R., and Khan, M. A. (2007). Controlled release multiparticulate beads coated with starch acetate: Material characterization, and identification of critical formulation and process variables. Pharmaceutical development and technology 12, 307-320.

Planas, M., Gonzalez, P., Rodriguez, L., Sanchez, S., and Cevc, G. (1992). Noninvasive percutaneous induction of topical analgesia by a new type of drug carrier, and prolongation of local pain insensitivity by anesthetic liposomes. Anesthesia \& Analgesia 75, 615-621.

Pohle, W., Gauger, D., Fritzsche, H., Rattay, B., Selle, C., Binder, H., and Böhlig, H. (2001). FTIR-spectroscopic characterization of phosphocholine-headgroup model compounds. Journal of molecular structure 563, 463-467.

Shen, L.-N., Zhang, Y.-T., Wang, Q., Xu, L., and Feng, N.-P. (2014). Enhanced in vitro and in vivo skin deposition of apigenin delivered using ethosomes. International journal of pharmaceutics 460, 280-288.

Sweetman, S. C. (2009). Martindale: the complete drug reference: Pharmaceutical press).

Touitou, E., Dayan, N., Bergelson, L., Godin, B., and Eliaz, M. (2000). Ethosomesnovel vesicular carriers for enhanced delivery: characterization and skin penetration properties. Journal of Controlled Release 65, 403-418.

Trotta, M., Peira, E., Carlotti, M. E., and Gallarate, M. (2004). Deformable liposomes for dermal administration of methotrexate. International journal of pharmaceutics 270, 119-125.

van den Bergh, B. A., Wertz, P. W., Junginger, H. E., and Bouwstra, J. A. (2001). Elasticity of vesicles assessed by electron spin resonance, electron microscopy and extrusion measurements. International journal of pharmaceutics $217,13-$ 24.

Westerberg, D. P., and Voyack, M. J. (2013). Onychomycosis: Current trends in diagnosis and treatment. American family physician 88.

Yang, Y., Ou, R., Guan, S., Ye, X., Hu, B., Zhang, Y., Lu, S., Zhou, Y., Yuan, Z., and Zhang, J. (2015). A novel drug delivery gel of terbinafine hydrochloride with high penetration for external use. Drug delivery 22, 1086-1093.

Zhang, J.-P., Wei, Y.-H., Zhou, Y., Li, Y.-Q., and Wu, X.-A. (2012). Ethosomes, binary ethosomes and transfersomes of terbinafine hydrochloride: a comparative study. Archives of pharmacal research 35, 109-117. 


\section{دراسة مقارنة بين تركيبات مختلفة لحويصلات النانولعقار التربينافين هياروكلوريد من خلال صياغات تتخلل عبر الجلا وتأثيرها المضاد للفئوريات \\ لانسادة الاكاتزة \\ علاء عبداله ذكى' محمود محمد توفيق '

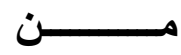

'قسم الصيدلانيات و الصيدلة الصناعية، كلية الصيدلة، جامعة الأزهر ، مدينة نصر ، القاهرة، مصر.

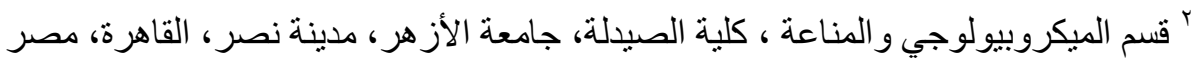

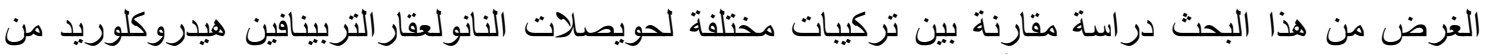

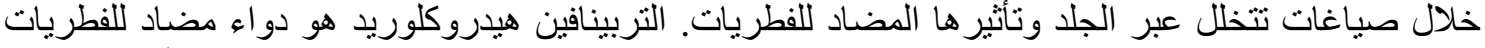

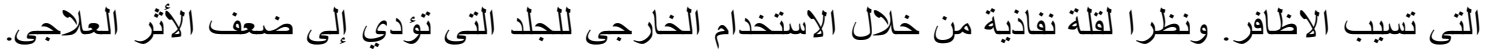

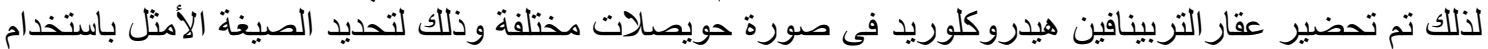

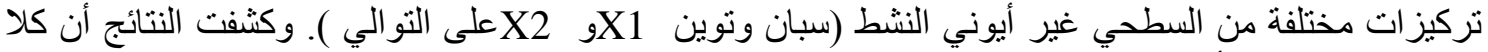

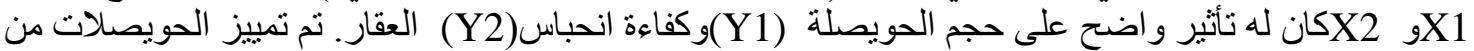

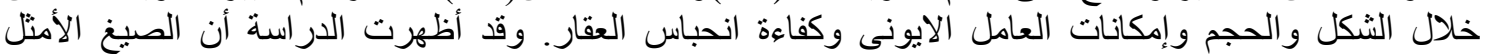

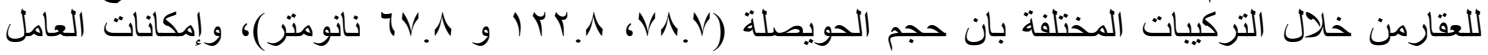

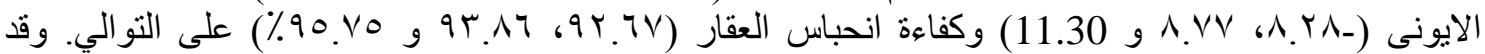

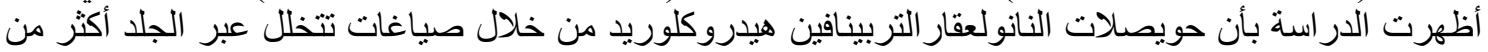

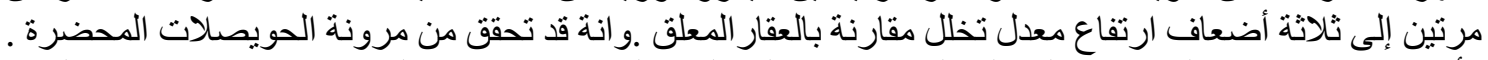

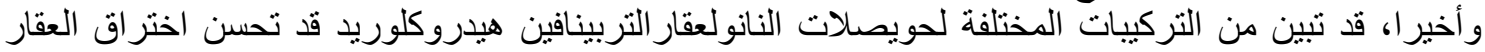

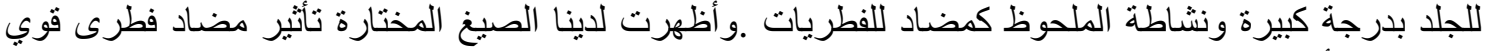
لعلاج فطر أظافر اليدين و القدمين. 